$\xi=-1$

\title{
Some characterizations of raised cosine distribution
}

\author{
M. Ahsanullah ${ }^{1}$ *, M. Shakil ${ }^{2}$ \\ ${ }^{1}$ Rider University, Lawrenceville, $N J$, USA \\ ${ }^{2}$ Miami Dade College, Hialeah, FL 33012, USA \\ * Corresponding authorE-mail: mshakil@mdc.edu
}

\begin{abstract}
Some distributional properties of the raised cosine distribution are presented. Based on the distributional properties, several new characterizations of the raised cosine distribution are given.
\end{abstract}

Keywords: Characterization; Raised Cosine Distribution; Truncated First Moment.

\section{Introduction}

Fitting of probability distributions to the real-world data is an important area of research in the fields of probability and statistics. However, before we fit a particular probability distribution to these data, it becomes necessary to justify whether the given probability distribution satisfies the underlying requirements by its characterization. Many authors and researchers have developed various methods for the characterizations of probability distributions, for which the interested readers are referred to Nagaraja [17], Ahsanullah et al. [4], Ahsanullah [2], and references therein. For example, in recent years, many researchers have investigated the characterizations of probability distributions based on a simple relationship between two different moments truncated from the left at the same point, see, for example, Galambos and Kotz [9], Kotz and Shanbhag [14], Glänzel et al. [11], and Glänzel [10], among others. As pointed out by Glänzel [10], these characterizations may serve as a basis for parameter estimation, and may also be useful in developing some goodness-of-fit tests of distributions by using data whether they satisfy certain properties given in the characterizations of distributions. For further discussions in this regard, the interested readers are referred to Ahsanullah et al. [56].

It appears from the literature that not much attention has been paid to the characterization of the raised cosine distribution. For details on the raised cosine distribution and its applications, the interested readers are referred to King [13], Rinne [21], and Willink [23], among others. As pointed out by Kyurkchiev and Kyurkchiev [15], the raised-cosine distribution function and raised-cosine cumulative distribution function are functions commonly used to avoid inter symbol interference in communications systems. For details, the interested readers are referred to their paper. In this paper, motivated by the importance of the raised cosine distribution in many practical problems, we consider its several distributional properties. Based on these distributional properties, we have established some new characterizations of the raised cosine distribution by truncated first moment, order statistics and upper record values, which, we hope, will be useful for practitioners and researchers in the fields of probability, statistics, and other applied sciences. The organization of this paper is as follows. Section 2 briefly discusses the raised cosine distribution and some of its properties. The main results on the characterizations of the raised cosine distribution are presented in section 3. The concluding remarks are provided in Section 4.

\section{Raised cosine distribution and its distribu- tional properties}

As pointed out above, for details on the raised cosine distribution and its applications, the interested readers are referred to King [13], Kyurkchiev and Kyurkchiev [15], Rinne [21], and Willink [23], among others. For the sake of completeness, here we briefly discuss some of the distributional properties of the raised cosine distribution.

A continuous random variable $X$ is said to have raised cosine distribution (RCD) if its probability density function (pdf) $f(x)$ is as follows:

$$
f(x, \mu, \sigma)=\frac{1}{2 \sigma}\left[1+\cos \left(\frac{\pi(x-\mu)}{\sigma}\right)\right],
$$

where $-\infty<\mu<\infty, \mu-\sigma<x<\mu+\sigma, \sigma>0$. It is easy to see that the mean of the raised cosine distribution (1) is given by

$$
\begin{aligned}
& \text { Mean }=E(X) \\
& =\int_{\mu-\sigma}^{\mu+\sigma} x \frac{1}{2 \sigma}\left[1+\cos \left(\frac{\pi(x-\mu)}{\sigma}\right)\right] d x=\mu .
\end{aligned}
$$

To describe the shapes of the raised cosine distribution (RCD), $X \sim R C D(\mu, \sigma)$, using Maple software, the plots of the pdf (1), for $\sigma=1,1.5,2.5,3,3.5$, when $\mu=0$, are provided below in Figure 1. 


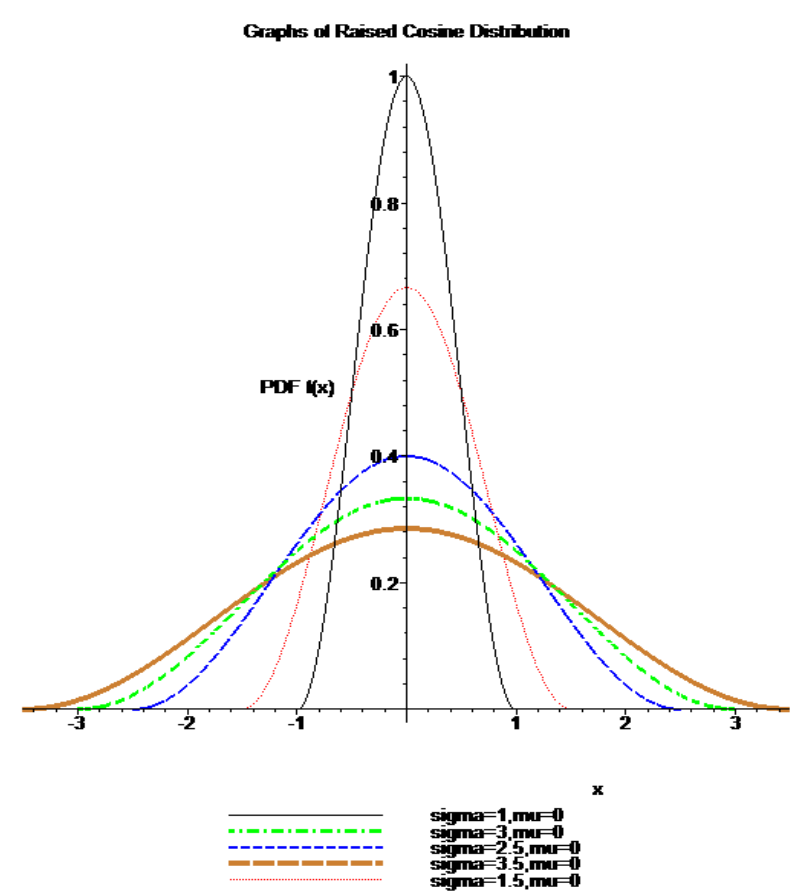

Fig. 1: $\operatorname{PDF} f(x)$ of $X \sim R C D(\mu, \sigma)$, for $\sigma=1,1.5,2.5,3,3.5$, when $\mu=0$.

The effects of the parameters can easily be seen from these graphs. We note that, since the raised cosine distribution,

$X \sim R C D(\mu, \sigma)$, is an even function, it is easily seen that the pdf of $X \sim R C D(\mu, \sigma)$ is symmetric about the mean $\mu=0$. Similarly, we can draw graphs of the pdf of $X \sim R C D(\mu, \sigma)$ for other values of the parameters. The corresponding cumulative distribution function (cdf) $F(x)$, reliability function $R(x)$, hazard function $h(x)$, and cumulative hazard function $H(x)$ are respectively given by

$$
\begin{aligned}
& F(x)=\frac{1}{2}\left[1+\frac{x-\mu}{\sigma}+\frac{1}{\pi} \sin \left(\frac{\pi(x-\mu)}{\sigma}\right)\right], \\
& R(x)=\frac{1}{2}\left[1-\frac{x-\mu}{\sigma}-\frac{1}{\pi} \sin \left(\frac{\pi(x-\mu)}{\sigma}\right)\right], \\
& h(x)=\frac{\left[1+\cos \left(\frac{\pi(x-\mu)}{\sigma}\right)\right]}{\sigma\left(1-\frac{x-\mu}{\sigma}-\frac{1}{\pi} \sin \left(\frac{\pi(x-\mu)}{\sigma}\right)\right)},
\end{aligned}
$$

and

$$
H(x)=-\ln (R(x))=\ln (2 \pi)-\ln \left[\pi\left(1-\frac{x-\mu}{\sigma}\right)-\sin \left(\frac{\pi(x-\mu)}{\sigma}\right)\right]
$$

Moment Generating Function: Using the equation (1) for the pdf $f(x, \mu, \sigma)$, the moment generating function $M_{X}(t)$ is given

$$
M_{X}(t)=\int_{\mu-\sigma}^{\mu+\sigma} e^{t x} \frac{1}{2 \sigma}\left[1+\cos \left(\frac{\pi(x-\mu)}{\sigma}\right)\right] d x,
$$

which on substituting $\frac{\pi(x-\mu)}{\sigma}=z$, and using the well-

known integral formula

$\int e^{a x} \cos b x d x=\frac{e^{a x}}{a^{2}+b^{2}}(a \cos b x+b \sin b x)+C$,

and noting that $\sinh (z)=\frac{1}{2}\left(e^{z}-e^{-z}\right)$, it is easy to see that,

after simplification, the moment generating function $M_{X}(t)$ is given by

$$
\begin{aligned}
M_{X}(t) & =\frac{1}{2} \frac{\pi^{2} e^{\mu t}\left(e^{\sigma t}-e^{-\sigma t}\right)}{\sigma t\left(\pi^{2}+\sigma^{2} t^{2}\right)} \\
& =\frac{\pi^{2} \sinh (\sigma t)}{\sigma t\left(\pi^{2}+\sigma^{2} t^{2}\right)} e^{\mu t}, 0<t<\frac{\pi}{\sigma} .
\end{aligned}
$$

Characteristic Function: Following in the same manner as above, the characteristic function $\Phi_{x}(t)$ is easily obtained as follows

$$
\begin{aligned}
\Phi_{x}(t) & =\int_{\mu-\sigma}^{\mu+\sigma} e^{i t x} \frac{1}{2 \sigma}\left[1+\cos \left(\frac{\pi(x-\mu)}{\sigma}\right)\right] d x=\frac{1}{2} \frac{\pi^{2} e^{i \mu t}\left(e^{i \sigma t}-e^{-i \sigma t}\right)}{i \sigma t\left(\pi^{2}+i^{2} \sigma^{2} t^{2}\right)} \\
& =\frac{\pi^{2} \sinh (i \sigma t)}{i \sigma t\left(\pi^{2}+i^{2} \sigma^{2} t^{2}\right)} e^{i \mu t}=\frac{\pi^{2} \sin (\sigma t)}{\sigma t\left(\pi^{2}-\sigma^{2} t^{2}\right)} e^{i \mu t},
\end{aligned}
$$

since $\sinh (i \sigma t)=-i \sin (i(i \sigma t))$, where $i=\sqrt{-1}$.

Variance: It is easy to see that the variance of the raised cosine distribution (1) is given by

$$
\begin{aligned}
\text { Variance }=E\left(X^{2}\right)-[E(X)]^{2} & =\frac{3 \pi^{2} \mu^{2}+\pi^{2} \sigma^{2}-6 \sigma^{2}}{3 \pi^{2}}-\mu^{2} \\
& =\sigma^{2}\left[\frac{1}{3}-\frac{2}{\pi^{2}}\right] .
\end{aligned}
$$

Median: Since the raised cosine distribution is symmetric about the mean $\mu$, its median $M D$ will also be given by $M D=\mu$, which can also be easily seen by solving the following equation for $x$ :

$$
F(x)=\frac{1}{2}\left[1+\frac{x-\mu}{\sigma}+\frac{1}{\pi} \sin \left(\frac{\pi(x-\mu)}{\sigma}\right)\right]=0.5 .
$$

Mode: The mode of the raised cosine distribution is the value of $x=x_{m}$ (say), for which its pdf (1), that is,

$$
f(x, \mu, \sigma)=\frac{1}{2 \sigma}\left[1+\cos \left(\frac{\pi(x-\mu)}{\sigma}\right)\right]
$$

is maximum. Now, differentiating the above equation for $f(x)$, we have

$$
\frac{d f(x)}{d x}=-\frac{\pi}{2 \sigma^{2}} \sin \left(\frac{\pi(x-\mu)}{\sigma}\right)
$$

which, when equated to 0 , and solving for $x$, easily gives $x=x_{m}=\mu$. It can be easily seen that 
$\frac{d^{2} f(x)}{d x^{2}}=-\frac{\pi^{2}}{2 \sigma^{3}} \cos \left(\frac{\pi(x-\mu)}{\sigma}\right)$ is $<0$, when

$x=x_{m}=\mu$, and thus the mode of the raised cosine distribution is $x_{m}=\mu$, and the maximum value of the pdf (1) is given by $f_{X}\left(x_{m}\right)=f_{X}(\mu)$. Clearly, the raised cosine distribution is unimodal.

Inflection Points: By solving the following equation

$$
\frac{d^{2} f(x)}{d x^{2}}=-\frac{\pi^{2}}{2 \sigma^{3}} \cos \left(\frac{\pi(x-\mu)}{\sigma}\right)=0
$$

for $x$, it can easily be seen that the inflection points are given by $x=\mu \pm \frac{\sigma}{2}$, since the pdf $f(x)$ given by Eq. (1) is concave up in the intervals $\left(\mu-\sigma, \mu-\frac{\sigma}{2}\right) \cup\left(\mu+\frac{\sigma}{2}, \mu+\sigma\right)$ and concave down in the interval $\left(\mu-\frac{\sigma}{2}, \mu+\frac{\sigma}{2}\right)$.

Standard Raised Cosine Distribution: The pdf $f(x)$ and the cdf $F(x)$ of the standard raised cosine distribution,

$X \sim R C D(0,1)$, are given as follows:

$f(x)=\frac{1}{2}[1+\cos (\pi x)]$,

and

$$
F(x)=\frac{1}{2}\left[1+x+\frac{1}{\pi} \sin (\pi x)\right] .
$$

Graphs: The graph of the pdf (2) of $X \sim R C D(0,1)$ is given in Figure 2.

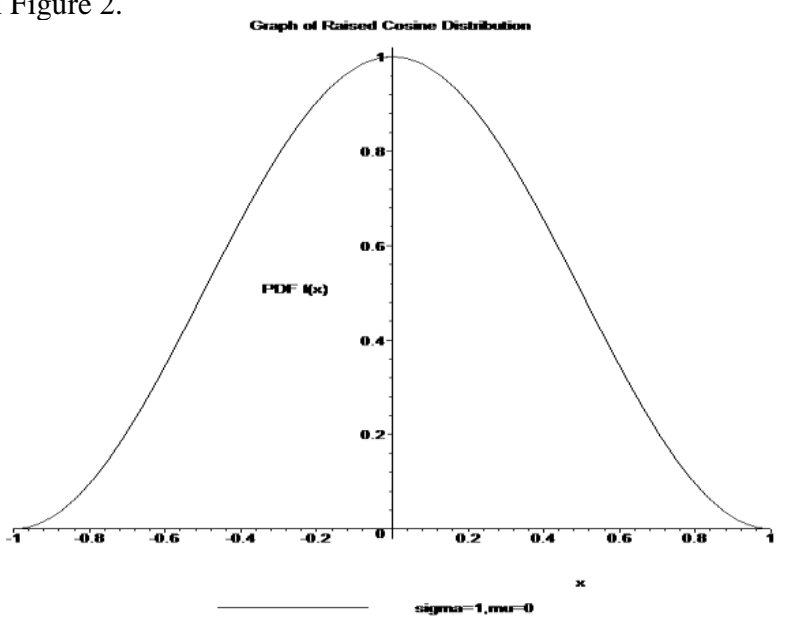

Figure 2: PDF $f(x)$ of $X \sim R C D(0,1)$.

We Observe that the Pdf of $X \sim R C D(0,1)$ is Symmetric around zero. The Graphs of the CDF $F(x)$, Reliability Function
$R(x)$, Hazard Function $h(x)$, and Cumulative Hazard Function $H(x)$ of $X \sim R C D(0,1)$ are given in the Following Figures $3-6$ respectively.

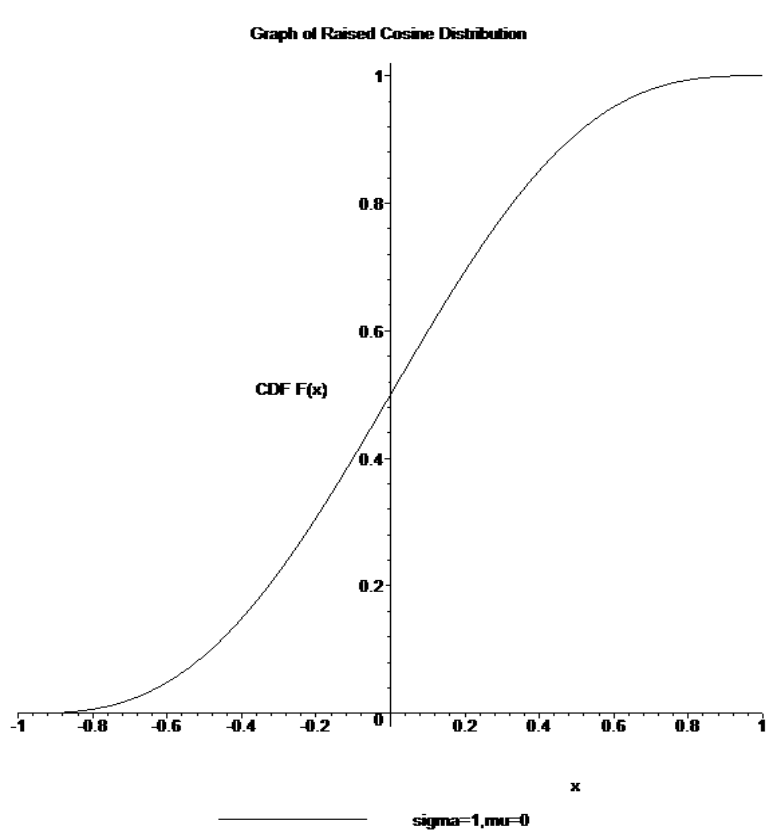

Figure 3: $\operatorname{CDF} F(x)$ of $X \sim R C D(0,1)$.

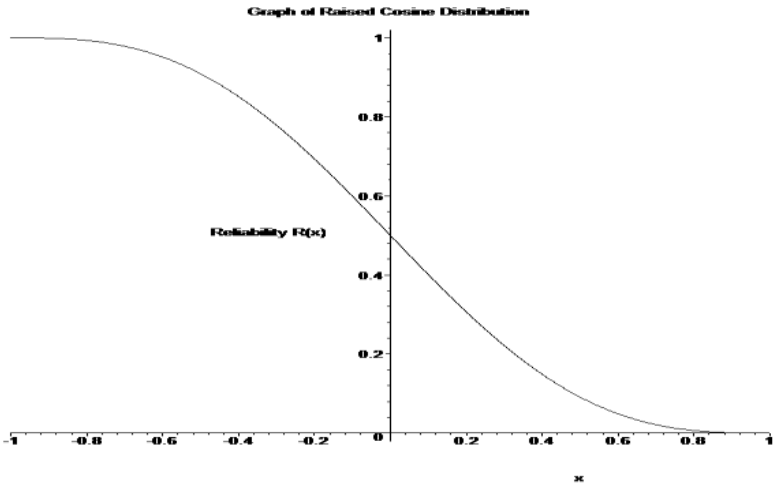

Figure 4: Reliability Function $R(x)$ of $X \sim R C D(0,1)$.

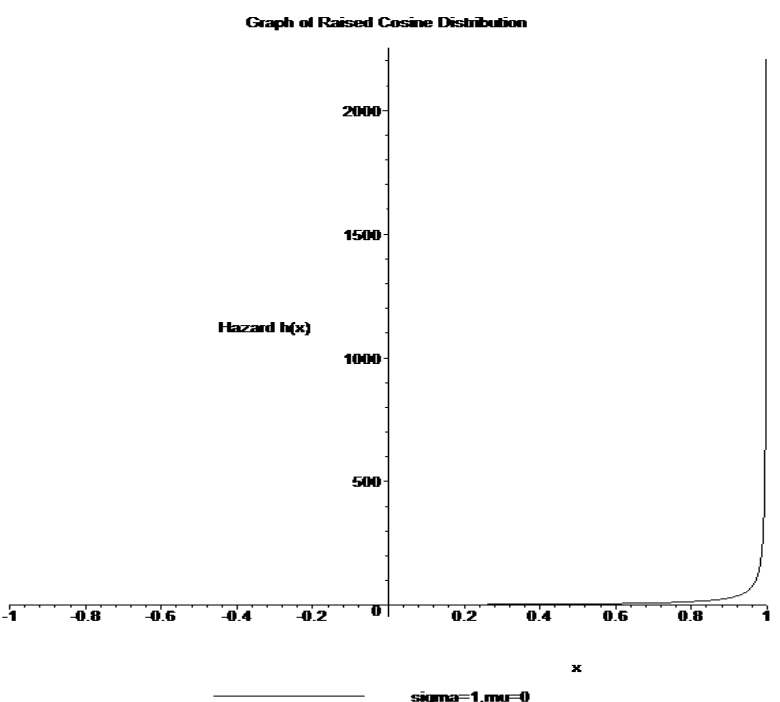

Figure 5: Hazard Function $h(x)$ of $X \sim R C D(0,1)$. 


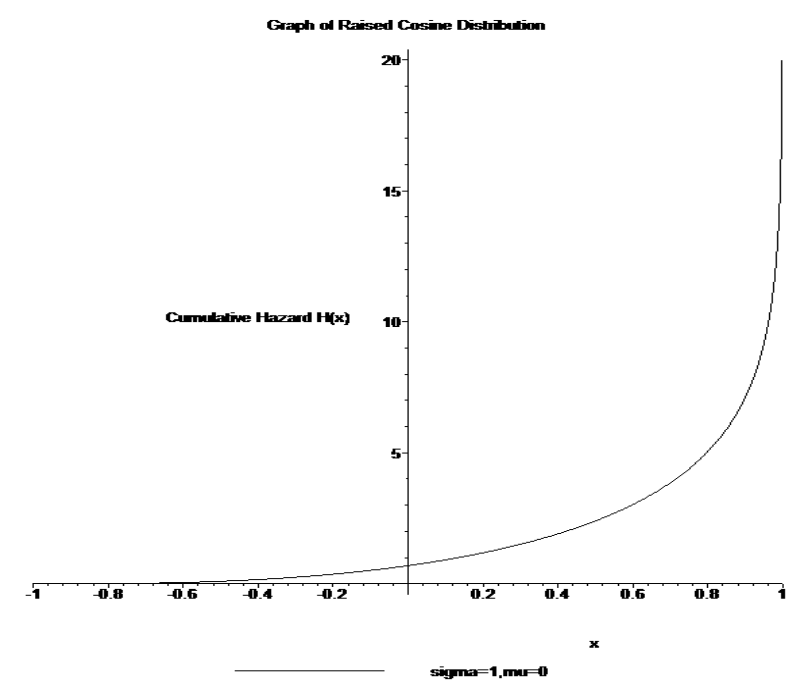

Fig. 6: Cumulative Hazard Function $H(x)$ of $X \sim \operatorname{RCD}(0,1)$.

Moments: Since the standard raised cosine distribution,

$X \sim \operatorname{RCD}(0,1)$, is an even function, it is easy to see that all odd moments of $X \sim R C D(0,1)$ are zero. For the even moments, when $n$ is a positive integer, using the pdf (2) of $X \sim \operatorname{RCD}(0,1)$, we have

$E\left(X^{2 n}\right)=\int_{-1}^{1} x^{2 n}\left[\frac{1}{2}[1+\cos (\pi x)]\right] d x$

which, on successive integration (that is, using reduction formula), is easily expressed as

$E\left(X^{2 n}\right)=\frac{1}{2 n+1}-\frac{1}{\pi^{2 n}} \sum_{j=1}^{n} \frac{(-1)^{j-1}(2 n) !(\pi)^{2 n-2 j}}{(2 n-(2 j-1)) !}$,

where $n=1,2,3, \ldots$, correspond to the even moments $E\left(X^{2}\right), E\left(X^{4}\right), E\left(X^{6}\right), \ldots$, respectively, which, by using the above equation, are easily computed as follows:

$$
\begin{aligned}
& E\left(X^{2}\right)=\frac{1}{3}-\frac{2}{\pi^{2}} \approx 0.13069, \\
& E\left(X^{4}\right)=\frac{1}{5}-\frac{1}{\pi^{4}}\left(4 \pi^{2}-24\right) \approx 0.04110, \\
& E\left(X^{6}\right)=\frac{1}{7}-\frac{1}{\pi^{6}}\left(6 \pi^{4}-120 \pi^{2}+720\right) \approx 0.01793 .
\end{aligned}
$$

Remarks: Also, following Gradshteyn and Ryzhik [12], and Prudnikov et al. [19], Vol. 1, Eq. 2.5.8.1, page 393, we easily obtain the following expressions for the event moment, $E\left(X^{2 n}\right)$ :

$$
\begin{aligned}
& E\left(X^{2 n}\right)=\int_{-1}^{1} x^{2 n}\left[\frac{1}{2}[1+\cos (\pi x)]\right] d x \\
& =\frac{1}{2 n+1}+\frac{1}{2 n+1} F_{2}\left(n+\frac{1}{2} ; \frac{1}{2}, n+\frac{3}{2} ;-\frac{\pi^{2}}{4}\right),
\end{aligned}
$$

where ${ }_{1} F_{2}\left(n+\frac{1}{2} ; \frac{1}{2}, n+\frac{3}{2} ;-\frac{\pi^{2}}{4}\right)$ is the generalized hypergeometric function defined by the equation

$$
{ }_{1} F_{2}(a ; b, c ; z)=\sum_{k=0}^{x} \frac{(a)_{k}}{(b)_{k}(c)_{k}} \frac{z^{k}}{k !},
$$

which is an absolutely convergent series for all real values of $z$, where $a, b, c$ are real numbers, and $(a)_{k}$ is the Pochhammer symbol (rising factorial) that is defined by $(a)_{0}=1$, $(a)_{k}=\frac{\Gamma(a+k)}{\Gamma(a)}=a(a+1)(a+2) \ldots(a+k-1)$, where $k \in \mathrm{N}$. For details on the generalized hypergeometric function ${ }_{1} F_{2}(a ; b, c ; z)$, see, for example, Gradshteyn and Ryzhik [12], Mathai and Saxena [16], Prudnikov et al. [20], and Slater [22], among others. For the sake of completeness, using the above expressions, the first ten even moments of the standard raised cosine distribution $X \sim R C D(0,1)$, are computed by using Maple software, which are provided in the Table 1 below.

Table 1: First Ten Even Moments of the Standard Raised Cosine Distribution, $X \sim R C D(0,1)$

\begin{tabular}{ll}
\hline$n$ & $E\left(X^{2 n}\right)$ \\
\hline 1 & 0.130691 \\
2 & 0.04110 \\
3 & 0.01793 \\
4 & 0.00937 \\
5 & 0.00550 \\
6 & 0.00349 \\
7 & 0.00235 \\
8 & 0.00166 \\
9 & 0.00121 \\
10 & 0.00091 \\
\hline
\end{tabular}

It's observed from Table 1 that the even moment $X \sim R C D(0,1)$ is a decreasing function of $n$.

Percentile Points: Here we compute the percentile points of the standard raised cosine distribution, $X \sim \operatorname{RCD}(0,1)$, with the $\operatorname{pdf}(2)$ and cdf (3). For any $0<p<1$, the $100 p$ th percentile (also called the quantile of order $p$ of the standard raised cosine distribution, $X \sim R C D(0,1)$, is a number $x_{p}$ such that the area under $f_{X}(x)$ to the left of $x_{p}$ is $p$. That is, $x_{p}$ is any root of the equation given by

$F\left(x_{p}\right)=\int_{-1}^{x_{p}} f_{X}(u) d u=p$. The percentile points $x_{p}$ associated with the cdf of $X \sim R C D(0,1)$, that is, $F(x)=\frac{1}{2}\left[1+x+\frac{1}{\pi} \sin (\pi x)\right]$, are computed by using

Maple software, which are provided in the Table 2 below. 
Table 2: Percentile Points of the Standard Raised Cosine Distribution, $X \sim R C D(0,1)$

\begin{tabular}{ll}
\hline$p$ & $x_{p}$ \\
\hline 0.1 & -0.48219 \\
0.2 & -0.32737 \\
0.3 & -0.20716 \\
0.4 & -0.10084 \\
0.5 & 0.0 \\
0.6 & 0.10084 \\
0.7 & 0.20716 \\
0.8 & 0.32737 \\
0.9 & 0.48219 \\
\hline
\end{tabular}

\section{Characterizations}

In this section, we present some new characterizations of the raised cosine distribution by truncated first moment, order statistics and upper record values. Without loss of generality, we will consider the standard raised cosine distribution,

$X \sim R C D(0,1)$, with the pdf (2) and cdf (3).

Assumption and Lemmas: In order to prove our main results on characterization, we will need the following assumption (Assumption 1), along with two lemmas, (Lemmas 1 and 2), which will be useful in proving our main results.

Assumption 1: Suppose the random variable $X$ is absolutely continuous with cumulative distribution function (cdf) $F(x)$ and probability density function (pdf) $f(x)$. We assume that $\gamma=\{x \mid F(x)>0\}$ and $\delta=\inf \{x \mid F(x)<1\}$. We further assume that $E(X)$ exists.

Lemma 1: Under the assumption 1, if $E(X \mid X \leq x)=g(x) \tau(x)$, where $\tau(x)=\frac{f(x)}{F(x)}$ and $g(x)$ is a continuous differentiable function of $x$ with the condition that $\int_{\gamma}^{x} \frac{u-g^{\prime}(u)}{g(u)} d u$ is finite for all $x, \gamma<x<\delta$, then $f(x)=c e^{\int_{\gamma}^{x} \frac{u-g^{\prime}(u)}{g(u)} d u}$, where $c$ is determined by the condition $\int_{\gamma}^{\delta} f(x) d x=1$.

Proof: We have $g(x)=\frac{\int_{\gamma}^{x} u f(u) d u}{f(x)}$, that is,

$\int_{\gamma}^{x} u f(u) d u=f(x) g(x)$.

Differentiating the above equation with respect to respect to $x$, we obtain

$x f(x)=f^{\prime}(x) g(x)+f(x) g^{\prime}(x)$

From the above equation, we obtain

$$
\frac{f^{\prime}(x)}{f(x)}=\frac{x-g^{\prime}(x)}{g(x)}
$$

On integrating the above equation with respect to $x$, we have $f(x)=c e^{\int_{\gamma}^{\gamma} \frac{u-g^{\prime}(u)}{g(u)} d u}$, where $c$ is obtain by the condition $\int_{\gamma}^{\delta} f(x) d x=1$. This completes the proof of Lemma 1 .

Lemma 2: Under the assumption 1, if $E(X \mid X \geq x)=g(x) r(x)$, where $r(x)=\frac{f(x)}{1-F(x)}$ and $g(x)$ is a continuous differentiable function of $x$ with the condition that $\int_{x}^{\delta} \frac{u+g^{\prime}(u)}{g(u)} d u$ is finite for all $x, \gamma<x<\delta$, then $f(x)=c e^{-\int_{\gamma}^{x} \frac{u+g^{\prime}(u)}{g(u)} d u}$, where $c$ is determined by the condition $\int_{\gamma}^{\delta} f(x) d x=1$.

Proof: We have $g(x)=\frac{\int_{x}^{\delta} u f(u) d u}{f(x)}$, that is, $\int_{x}^{\delta} u f(u) d u=f(x) g(x)$

Differentiating the above equation with respect to respect to $x$ we obtain

$-x f(x)=f^{\prime}(x) g(x)+f(x) g^{\prime}(x)$

From the above equation, we obtain

$$
\frac{f^{\prime}(x)}{f(x)}=-\frac{x+g^{\prime}(x)}{g(x)} \text {. }
$$

On integrating the above equation with respect to $x$, we have

$f(x)=c e^{-\int^{x} \frac{u+g^{\prime}(u)}{g(u)} d u}$

where $c$ is obtained by the condition $\int_{\gamma}^{\delta} f(x) d x=1$. This completes the proof of Lemma 2.

Main Results: Here, we establish our main results in Theorems 1 5 .

Characterization by Truncated First Moment: The following two theorems are based on truncated first moment.

Theorem 1: Suppose that $X$ is an absolutely continuous random variable with cdf $F(x)$ with $F(-1)=0, F(1)=1$, and $E(X)$ exists. Then $E(X \mid X \leq x)=g(x) \tau(x)$, where $g(x)=\frac{\frac{x^{2}-1}{2}+\frac{x}{\pi} \sin (\pi x)+\frac{1}{\pi^{2}}[1+\cos (\pi x)]}{1+\cos (\pi x)}$, and $\tau(x)=\frac{f(x)}{F(x)}$, if and only if $f(x)=\frac{1}{2}[1+\cos (\pi x)]$, which is the pdf of the standard raised cosine distribution, $X \sim R C D(0,1)$. 
Proof: Since $E(X \mid X \leq x)=\frac{\int_{\gamma}^{x} \theta f(\theta) d \theta}{F(x)}$ and

$\tau(x)=\frac{f(x)}{F(x)}$, we have $g(x)=\frac{\int_{\gamma}^{x} \theta f(\theta) d \theta}{f(x)}$. Now, if

the random variable $X$ satisfies the Assumption 1 and has the standard raised cosine distribution, that is,

$$
f(x)=\frac{1}{2}[1+\cos (\pi x)]
$$

then, after simplification, we have

$$
\begin{aligned}
g(x) & =\frac{\int_{-1}^{x} \theta f(\theta) d \theta}{f(x)}=\frac{\int_{-1}^{x} \theta(1+\cos \pi \theta) d \theta}{1+\cos (\pi x)} \\
& =\frac{\frac{x^{2}-1}{2}+\frac{x}{\pi} \sin (\pi x)+\frac{1}{\pi^{2}}[1+\cos (\pi x)]}{1+\cos (\pi x)} .
\end{aligned}
$$

Consequently, the proof of "if" part of the Theorem 1 follows from Lemma 1.

Conversely, we will now prove the "only if" condition of Theorem 1. Suppose that

$$
g(x)=\frac{\frac{x^{2}-1}{2}+\frac{x}{\pi} \sin (\pi x)+\frac{1}{\pi^{2}}[1+\cos (\pi x)]}{1+\cos (\pi x)},
$$

from which, after differentiation, we easily have

$$
g^{\prime}(x)=x+g(x) \frac{\pi \sin (\pi x)}{1+\cos (\pi x)},
$$

or,

$$
\frac{x-g^{\prime}(x)}{g(x)}=-\frac{\pi \sin (\pi x)}{1+\cos (\pi x)} .
$$

Consequently, by using Lemma 1, we obtain

$$
\frac{f^{\prime}(x)}{f(x)}=\frac{x-g^{\prime}(x)}{g(x)}=-\frac{\pi \sin (\pi x)}{1+\cos (\pi x)} .
$$

On integrating the above equation (4) with respect to $x$, we obtain $f(x)=c(1+\cos (\pi x)$, where $c$ is a constant to be determined. Using the boundary conditions $F(-1)=0$ and $F(1)=1$, we have $c=\frac{1}{2}$, and thus $f(x)=\frac{1}{2}[1+\cos (\pi x)]$, which is the pdf of the standard raised cosine distribution, $X \sim R C D(0,1)$. This completes the proof of Theorem 1.

Theorem 2: If the random variable $X$ satisfies the Assumption 1 with $\gamma=-1$ and $\delta=1$, then

$E(X \mid X \geq x)=h(x) r(x)$, where $r(x)=\frac{f(x)}{1-F(x)}$

and $h(x)=\frac{\frac{1-x^{2}}{2}-\frac{x}{\pi} \sin (\pi x)-\frac{1}{\pi^{2}}[1+\cos (\pi x)]}{1+\cos (\pi x)}$,

if and only if $f(x)=\frac{1}{2}[1+\cos (\pi x)]$, which is the pdf of the standard raised cosine distribution, $X \sim R C D(0,1)$.

Proof: Suppose that $E(X \mid X \geq x)=h(x) r(x)$. Then,

since $E(X \mid X \geq x)=\frac{\int_{x}^{\delta} \theta f(\theta) d \theta}{1-F(x)}$ and

$r(x)=\frac{f(x)}{1-F(x)}$, we have $h(x)=\frac{\int_{x}^{\delta} \theta f(\theta) d \theta}{f(x)}$. Now,

if the random variable $X$ satisfies the Assumption 1 and has the standard raised cosine distribution, that is,

$f(x)=\frac{1}{2}[1+\cos (\pi x)]$

then, after simplification, we have

$$
h(x)=\frac{\int_{x}^{1} \theta f(\theta) d \theta}{f(x)}=\frac{\int_{x}^{1} \theta(1+\cos \pi \theta) d \theta}{1+\cos (\pi x)}
$$

$$
=\frac{\frac{1-x^{2}}{2}-\frac{x}{\pi} \sin (\pi x)-\frac{1}{\pi^{2}}[1+\cos (\pi x)]}{1+\cos (\pi x)} .
$$

Consequently, the proof of "if" part of the Theorem 2 follows from Lemma 2.

Conversely, we will now prove the "only if" condition of Theorem 2. Suppose that

$h(x)=\frac{\frac{1-x^{2}}{2}-\frac{x}{\pi} \sin (\pi x)-\frac{1}{\pi^{2}}[1+\cos (\pi x)]}{1+\cos (\pi x)}$,

from which, after differentiation, we easily have 


$$
h^{\prime}(x)=-x+h(x) \frac{\pi \sin (\pi x)}{1+\cos (\pi x)}
$$

or,

$$
\frac{x+h^{\prime}(x)}{h(x)}=\frac{\pi \sin (\pi x)}{1+\cos (\pi x)}
$$

Consequently, by using Lemma 2, we obtain

$$
\frac{f^{\prime}(x)}{f(x)}=-\frac{x+h^{\prime}(x)}{h(x)}=-\frac{\pi \sin (\pi x)}{1+\cos (\pi x)} \text {. }
$$

On integrating the above equation (5) with respect to $x$, we obtain $f(x)=c(1+\cos (\pi x)$, where $c$ is a constant to be determined. Using the boundary conditions $F(-1)=0$ and $F(1)=1$, we have $c=\frac{1}{2}$, and thus $f(x)=\frac{1}{2}[1+\cos (\pi x)]$, which is the pdf of the standard raised cosine distribution, $X \sim R C D(0,1)$. This completes the proof of Theorem 2 .

Characterizations by Order Statistics: Here, we will provide the characterizations based on order statistics, for which we first recall the following well-known results.

Let $X_{1}, X_{2}, \ldots, X_{n}$ be $n$ independent copies of the random variable $X$ having absolutely continuous distribution function $F(x)$ and pdf $f(x)$. Suppose that $X_{1, n} \leq X_{2, n} \leq \ldots \leq X_{n, n}$ are the corresponding order statistics. It is known that $X_{j, n} \mid X_{k, n}=x$, for $1 \leq k<j \leq n$, is distributed as the $(j-k)$ th order statistics from $(n-k)$ independent observations from the random variable $V$ having the pdf $f_{V}(v \mid x)$ where $f_{V}(v \mid x)=\frac{f(v)}{1-F(x)}, 0 \leq v<x$, see, for example, Ahsanullah et al. [3], chapter 5, or Arnold et al. [7], chapter 2, among others. Further, $X_{i, n} \mid X_{k, n}=x, 1 \leq i<k \leq n$, is distributed as $i t h$ order statistics from $k$ independent observations from the random variable $W$ having the pdf $f_{W}(w \mid x)$ where $f_{W}(w \mid x)=\frac{f(w)}{F(x)}, w<x$. Let $S_{k-1}=\frac{1}{k-1}\left(X_{1, n}+X_{2, n}+\ldots+X_{k-1, n}\right)$, and $T_{k, n}=\frac{1}{n-k}\left(X_{k+1, n}+X_{k+2, n}+\ldots+X_{n \cdot n}\right)$.

In the following two theorems, we will provide the characterizations of the standard raised cosine distribution, $X \sim R C D(0,1)$, based on order statistics. Without loss of generality, we will consider the pdf (2) of the standard raised cosine distribution, that is,

$$
f(x)=\frac{1}{2}[1+\cos (\pi x)] .
$$

Theorem 3: Suppose the random variable $X$ satisfies the Assumption 1 with $\gamma=-1$ and $\delta=1$, then

$E\left(S_{k-1} \mid X_{k, n}=x\right)=g(x) \tau(x)$, where $\tau(x)=\frac{f(x)}{F(x)}$

and $g(x)=\frac{\frac{x^{2}-1}{2}+\frac{x}{\pi} \sin (\pi x)+\frac{1}{\pi^{2}}[1+\cos (\pi x)]}{1+\cos (\pi x)}$,

if and only if $f(x)=\frac{1}{2}[1+\cos (\pi x)]$.

Proof: It is known, see Ahsanullah et al. [3], and David and Nagaraja [8], that

$$
E\left(S_{k-1} \mid X_{k, n}=x\right)=E(X \mid X \leq x)
$$

Thus the result follows from Theorem 1.

Theorem 4: Suppose the random variable $X$ satisfies the Assumption 1 with $\gamma=-1$ and $\delta=1$, then $E\left(T_{k, n} \mid X_{k, n}=x\right)=h(x) r(x)$, where $r(x)=\frac{f(x)}{1-F(x)}$ and $h(x)=\frac{\frac{1-x^{2}}{2}-\frac{x}{\pi} \sin (\pi x)-\frac{1}{\pi^{2}}[1+\cos (\pi x)]}{1+\cos (\pi x)}$, if and only if $f(x)=\frac{1}{2}[1+\cos (\pi x)]$.

Proof: It is known, see Ahsanullah et al. [3], and David and Nagaraja [8], that

$$
E\left(T_{k, n} \mid X_{k, n}=x\right)=E(X \mid X \geq x)
$$

Thus the result follows from Theorem 2.

Characterization by Upper Record Values: Here, we will provide the characterizations based on upper record values, for which we first recall the following definitions. Suppose that $X_{1}, X_{2}, \ldots$ is a sequence of independent and identically distributed absolutely continuous random variables with distribution function $F(x)$ and pdf $f(x)$. Let $Y_{n}=\max \left(X_{1}, X_{2}, \ldots, X_{n}\right)$ for $n \geq 1$. We say that $X_{j}$ is an upper record value of $\left\{X_{n}, n \geq 1\right\}$ if $Y_{j}>Y_{j-1}, j>1$. The indices at which the upper records occur are given by the record times $\left\{U(n)>\min \left(j \mid j>U(n+1), X_{j}>X_{U(n-1)}, n>1\right)\right\}$ and $U(1)=1$. We will denote the $n t h$ upper record value as $X(n)=X_{U(n)}$. For details, see for example Ahsanullah [1], among others. 
In the following theorem, we will provide the characterization of the standard raised cosine distribution, $X \sim R C D(0,1)$,

based on upper record values. Without loss of generality, we will consider the pdf (2) of the standard raised cosine distribution, that is,

$$
f(x)=\frac{1}{2}[1+\cos (\pi x)] .
$$

Theorem 5: Suppose the random variable $X$ satisfies the Assumption 1 with $\gamma=-1$ and $\delta=1$, then

$$
E(X(n+1) \mid X(n)=x)=h(x) r(x) \text {, where }
$$$$
r(x)=\frac{f(x)}{1-F(x)} \text { and }
$$$$
h(x)=\frac{\frac{1-x^{2}}{2}-\frac{x}{\pi} \sin (\pi x)-\frac{1}{\pi^{2}}[1+\cos (\pi x)]}{1+\cos (\pi x)}, \text { if }
$$

and only if $f(x)=\frac{1}{2}[1+\cos (\pi x)]$.

Proof: It is known, see Ahsanullah et al. [3], and Nevzorov [18], that

$$
E(X(n+1) \mid X(n)=x)=E(X \mid X \geq x) .
$$

Thus the result follows from Theorem 2 .

\section{Concluding remarks}

A probability distribution can be characterized through various methods. In this paper, we present some new characterizations of the raised cosine distribution by truncated first moment, order statistics and upper record values. It is hoped that the findings of the paper will be useful for researchers in the fields of probability, statistics, and other applied sciences.

\section{Acknowledgement}

The authors are thankful to the referees for their valuable comments and suggestions, which certainly improved the presentation of the paper. The authors are also thankful to all those authors and researchers whose works were consulted during the preparation of this research paper, which have been properly cited here. Also, the second author, M. Shakil, is grateful to Miami Dade College for giving him the opportunity to be of service to this institution, without which it would have been impossible to conduct his research.

\section{References}

[1] Ahsanullah, M. (1995). Record Statistics, Nova Science Publishers, New York, USA

[2] Ahsanullah, M. (2017). Characterizations of Univariate Continuous Distributions, Atlantis-Press, Paris, France.

[3] Ahsanullah, M., Nevzorov,V. B., and Shakil, M. (2013). An Introduction to Order Statistics, Atlantis-Press, Paris, France. https://doi.org/10.2991/978-94-91216-83-1.

[4] Ahsanullah, M., Kibria, B. M. G., and Shakil, M. (2014). Normal and Student's t Distributions and Their Applications, Atlantis Press, Paris, France. https://doi.org/10.2991/978-94-6239-061-4.

[5] Ahsanullah, M., Shakil, M., and Kibria, B. G. (2015). Characterizations of folded student's t distribution. Journal of Statistical Distributions and Applications, 2(1), 15. https://doi.org/10.1186/s40488015-0037-5.

[6] Ahsanullah, M., Shakil, M., \& Kibria, B. M. (2016). Characterizations of Continuous Distributions by Truncated Moment. Journal of Modern Applied Statistical Methods, 15(1) 17 https://doi.org/10.22237/jmasm/1462076160.
[7] Arnold, B.C., Balakrishnan, and Nagaraja, H. N. (2005). First Course in Order Statistics, Wiley, New York, USA

[8] David, H. A., and Nagaraja, H. N. (2003). Order Statistics, Third Edition, Wiley, New York, USA https://doi.org/10.1002/0471722162.

[9] Galambos, J., and Kotz, S. (1978). Characterizations of probability distributions. A unified approach with an emphasis on exponential and related models, Lecture Notes in Mathematics, 675, Springer, Berlin

[10] Glänzel, W. (1987). A characterization theorem based on truncated moments and its application to some distribution families. Mathematical Statistics and Probability Theory (Bad Tatzmannsdorf, 1986), Vol. B, Reidel, Dordrecht, $75-84$ https://doi.org/10.1007/978-94-009-3965-3_8.

[11] Glänzel, W., Telcs, A., and Schubert, A. (1984). Characterization by truncated moments and its application to Pearson-type distributions. Z. Wahrsch. Verw. Gebiete, 66, 173-183.

[12] Gradshteyn, I. S., and Ryzhik, I. M. (1990). Table of integrals, series, and products, Academic Press, Inc., San Diego, California, USA.

[13] King, M. (2017). Statistics for Process Control Engineers: A Practical Approach, First Edition, Wiley, New York, USA. https://doi.org/10.1002/9781119383536.

[14] Kotz, S., and Shanbhag, D. N. (1980). Some new approaches to probability distributions. Advances in Applied Probability, 12, 903 921. https://doi.org/10.2307/1426748.

[15] Kyurkchiev, V., and Kyurkchiev, N. (2016). On the approximation of the step function by raised-cosine and laplace cumulative distribution functions. European International Journal of Science and Technology, 4(9), 75 - 84.

[16] Mathai, A. M. and Saxena, R. K. (1973). Generalized Hypergeometric Functions with ApplicationsIn Statistics and Physical Sciences, Lecture Notes No. 348. Springer-Verlag, Heidelberg, Germany.

[17] Nagaraja, H. (2006). Characterizations of Probability Distributions. In Springer Handbook of Engineering Statistics (pp. 79 - 95), Springer, London, UK. https://doi.org/10.1007/978-1-84628-28814.

[18] Nevzorov, V. B. (2001). Records: Mathematical Theory, Translation of Mathematical Monograph. American Mathematical Society, Rhode Island, USA.

[19] Prudnkov, A. P., Brychkov, Yu. A., and Marichev, O. I. (1986). Integrals and Series, Vol. 1, Gordon and Breach, New York, USA.

[20] Prudnkov, A. P., Brychkov, Yu. A., and Marichev, O. I. (1989). Integrals and Series, Vol. 3: More Special Functions, Gordon and Breach, New York, USA.

[21] Rinne, H. (2010). Location-Scale Distribution: Linear Estimation and Probability Plotting Using MATLAB, Copyright: Prof. em. Dr Hors Rinne, Department of Economics and Management Science, Justus-Lieblig-University, Giessen, Germany.

[22] Slater, L. J. (1966). Generalized Hypergeometric Functions, Cambridge University Press, Cambridge, England, UK.

[23] Willink, R. (2013). Measurement Uncertainty and Probability, Cambridge University Press, First Edition, New York, USA. https://doi.org/10.1017/CBO9781139135085. 